\title{
XVIII.
}

Aus dem Kaiserin Elisabeth-Wöchnerinnenheim "Lucina" in Wien Primararzt: Dr. Bosse.

\section{Zur Kenntnis der congenitalen Sacraltumoren}

\author{
Von \\ Dr. Kurt Frank \\ gew. Hausarzt des Institutes.
}

(Mit 2 Abbildungen.)

Seit jeher steht die Frage der angeborenen Sacraltumoren im Mittelpunkte des lebhaftesten Interesses. Es ist namentlich in den letzten Jahren die Casuistik dieser Geschwulstbildungen besonders durch Publikationen deutscher Autoren zu einer ganz stattlichen Zahl angewachsen. Der klinische Teil tritt begreiflicherweise hier vor dem anatomischen und entwicklungsgeschichtlichen Gesichtspunkte weit in den Hintergrund. Die Entstehungsweise der congenitalen Kreuzsteißbeingesehwülste ist bis auf den beutigen Tag noch der Gegenstand einer sehr gegensätzlichen Meinungsverschiedenheit. Es sind zwei unvereinbarliche Ansichten, die einander gegenüberstehen, die monound bigerminale Theorie.

Braune in Leipzig war der erste, der im Jabre 1868 eine zusammenfassende Darstellung der Sacraltumoren auf Grund makroskopischer Beobachtung gab. Später haben dann Virchow, Middeldorpf, Ritschelt, Nasse, v. Bergmann, Linser, und in jüngster Zeit Steinthal, Kiderlen, Borst, Wieting und Engelmann Beiträge zu dieser Frage geliefert. Alle diese genannten Forscher sind der Meinung, daß man bei den meisten Sacraltumoren mit der Annahme eines einzigen Keimes hinlänglich auskomme. Auch Lebert, der die Bezeichnung Dermoidcyste einführte, hatte 1852 von ähnlichen Voraussetzungen ausgehend, das „Gesetz der plastischen Heterotopie" aufgestellt, wonach sich einfache und zusammengesetzte Gewebe, ja selbst Organe infolge einer besonderen Ernährungsstörung an Stellen des Körpers bilden können, wo sie normalerweise nicht vorkommen. Nun ist diese Theorie mitsamt der Voraus- 
setzung eines eigentümlichen Nisus formativus wohl als überwunden anzusehen. Die monogerminale Theorie sieht jetzt in der überwiegenden Mehrheit der angeborenen Sacralgeschwülste eine Bildungshemmung normal angelegter und bereits differenzierter Organteile; es bestehe eine mangelhafte Rückbildung und gleichzeitig eine geschwulstartige Wucherung der gerade am unteren Stammesende zusammentreffenden Derivate aller 3 Keimblätter. Es wurden mit Zugrundelegung der entwicklungsgeschichtlichen Vorgänge die verschiedensten und absonderlichsten Ansichten über die engere Aetiologie der Sacraltumoren ausgesprochen. Middeldorpf sieht in dem postanalen Darmabschnitt, den Kowalewsky als Canalis neuroëntericus bezeichnete, die Ursprungsstätte dieser Tumoren und findet hiermit die Erklärung für das bäufige Vorkommen von Darmteilen, die einfach durch das Persistieren und die Weiterentwicklung dieses embryonalen Gebildes entstehen. Tourneux und Herman sprechen slch für die Entstehung aus den Vestiges coceygiennes-médul. laires aus. Auch die von Luschka entdeckte Steißdrüse wurde beschuldigt, durch Umwandlung angeborene Sacraltumoren hervorzurufen.

Meckel erklärte das Vorkommen von Gehirnsubstanz in den Tumoren durch die Annahme, es könne das untere Ende des Medullarrobres unter besonderen Umständen ebenso wie sonst das obere Ende zu einem Gehirn auswachsen. So geistreich diese Hypothese sein mag, so kann sie wohl kaum Anspruch auf besondere Glaubwürdigkeit erheben. Dennoch hat B orst vor nicht langer Zeit diese Ansicht für einen seiner Fälle ernstlich ins Treffen geführt.

Die meisten der genannten Autoren geben nun zu, daß man nicht bei allen Sacraltumoren mit der Annahme eines einzigen Keimes sein Auslangen finden könne und für die hochentwickelten, bereits zu den Doppelbildungen hinüberführenden Gebilden die bigerminale Theorie schwer entbehren könne.

Auch bier sind in jüngster Zeit einige Autoren, wie Kiderlen, namentlich aber Wieting und nach ihm Engelmann in ibrem allzu eifrigen Bestreben nach einer einheitlichen Genese so weit gegangen, daß sie decidiert die monogerminale Entstebung für die ausschließlich mögliche ansehen. Wieting und Engelmann vertreten den Standpunkt, daß nicht nur die einfach zusammengesetzten sondern auch die komplizierter gebauten Sacraltumoren aus Resten der foetalen Medullaranlage herzuleiten sind, die mit gleichzeitig verlagerten Teilen anderer Keimblätter in Wucherung geraten.

Kiderlen meint, die Annahme eines includierten Foetus sei 
zwar sehr bequem, aber gerade deshalb durchaus unhaltbar, da sie mit den natürlichen Vorstellungen und den tatsächlichen Verhältnissen im Widerspruch stehe. Dazu bemerkt $\mathrm{Hagen}$ in treffender Weise: „Es ist entschieden genialer und läßt einen höheren Gesichtspunkt erkennen, wenn man versucht, nicht allein die Sacralgeschwülste, sondern auch die anderwärts vorkommenden, aus Abkömmligen der 3 Keimblätter zusammengesetzten Tumoren in ihrer anscheinend oft hochgradigen Verschiedenheit zu einer Gruppe zu vereinigen und allen eine gemeinsame Genese zu gewährleisten, ein Versuch, der nur auf bigerminalem Wege Aussicht auf Erfolg verspricht."

Es ist auch nicht immer gar so leicht, wie z. B. bei den einfachen Tumoren die Annahme eines 2. Keimes konsequent und plausibel aufrecht zu erhalten. Man wird hier gewib gut daran tun, nicht blind zu generalisieren und etwa auch die simpelsten Dermoidcysten bigerminal erklären zu wollen, für welche Remak, Heschl und Thiersch in überzeugender Weise nachgewiesen haben, daß sie durch Abschnürung von Teilen des Ektoderms während des foetalen Lebens und durch Einschließung in andere Gewebe entstehen. Es hieße aber andrerseits sicherlich den Tatsachen Gewalt antun, wollte man die hochentwickelten Tumoren durch eine einzige Keimanlage erklären, selbst wenn ihre organähnlichen Gebilde nicht zu vollendeten Organsystemen zusammentreten. Es wurde seiner Zeit von Nasse die Behauptung aufgestellt, bei einer wirklichen foetalen Inclusion müßten Organe oder wenigstens erkennbare Reste von solchen Organen vorhanden sein, welche nicht von dem hinteren Leibesende des Embryo gebildet werden und daher nicht auf Keimversprengung, foetale Abschnürung oder abnorme Entwicklung zurückzuführen sind, die sich bei normaler Entwicklung zurückbilden und versehwinden. Ich habe bereits oben erwähnt, daß man auch auf dem bigerminalen Wege nicht zu weit gehen darf, doch wird sich in der Epikrise noch Gelegenheit bieten, die obige Behauptung auf ihr richtiges Maß einzuschränken.

Seit den grundlegenden Arbeiten Marchands und Bonnets hat man die Unterscheidung zwischen "Keimdrüsenteratomen" und den anderen im Körper vorkommenden Intrafoetationen endgiltig aufgegeben, womit auch die angeborenen Sacraltumoren aufgehört haben, als Geschwülste sui generis zu figurieren, sie stellen eben nur eine besondere Lokalisation dar.

Wilms, der sich um die Klarlegung dieses noch so dunklen, vielumstrittenen Gebietes große Verdienste erworben luat, hat die zu allgemein gehaltene Bezeichnung Sacraltumor ebenso wie den mysti- 
schen Namen "Teratom" fallen gelassen und an deren Stelle den Sammelnamen „Embryom" eingeführt. Gegen diese Bezeichnung, die von vielen Seiten akzeptiert wurde, hat sich besonders Saxer mit dem Bemerken gewendet, daß in diesen Tumoren fast nie embryonales Gewebe, sondern meist ausgebildete Gewebsformationen vorkommen.

Mit dem Namen "Embryom" soll aber nichts anderes als die häufigste Aetiologie dieser Geschwülste bezeichnet werden. Hagen bemerkt sehr richtig, daß diese Benennung zweifellos besser sei als Teratom, denn die Medizin ist heutzutage doch schon etwas exakter geworden, so daß die "wunderbaren Gebilde" eben beim Fortschreiten der Wissenschaft schon besser erkannt sind. Wilms hebt nachdrücklich hervor, daß es zur Aufklärung dieser Frage unbedingt nötig sei, alle Übergänge von den vollkommenen Formen bis zu den ganz rudimentären Einschlüssen nachzuweisen. Wenn nun Borst behauptet, in der Literatur diese Übergänge zwischen foetalen Inklusionen und den aus einem Keime abgeleiteten Sacraltumoren gefunden zu haben, so kann man bei genauer Überprüfung ihm ebensowenig wie Wieting Recht geben, der die neueren Publikationen uberhaupt nicht berücksichtigte. Aus all dem Gesagten geht wohl zur Genüge hervor, daß ein jeder neue, genau untersuchte Fall eine willkommene Bereicherung für diese Frage bilden muß, weshalb ich in den nachfolgenden Zeilen einen derartigen interessanten Fall mir zu publizieren erlaube, welchen ich im $\mathrm{K}$ a is erin Elisabeth Wöchnerinnenheım "Lucina" zu beobachten Gelegenheit hatte. Für die Ǘberlassung des Falles spreche ich Herrn Primarius Dr. Bosse meinen besten Dank aus.

Eigene Beobachtung.

J. F'., 23 Jahre alt, am 10. August 1903 aufgenommen. J.No. 308/190:3, amnormalen Schwangerschaftszustande. Parturiens ist immer gesund gewesen, in der Familie bisher keine Mißbildung beobachtet, menstruiert seit dem 16. Lebensjahre; Menses regelmäßig, reichlich und schmerzfrei. Vor 3 Jahren ein normaler Partus. Kind zeigte keine Besonderheiten, lebt und ist gesund. Letzte Menses Anfang November 1902.

Bei der Aufnahme erweisen sich die inneren Organe normal. Uterustumors längsoval, der 2 Querfinger unter dem Proc. xiph. endigt. Die Frucht befindet sich in S:chädellage II. Position und zeigt normale Herzaktion; der Schädel ist fix und mit einem kleinen Segment ins Becken eingetreten. Touchierbefund: Äußerer Muttermund für 2 Finger durchgängig. Cervicalkanal $4 \mathrm{~cm}$ lang. Innerer Muttermund geschlossen. Becken geräumig. Sp. 25 ; Cr. 27 ; Tr. 30. 7 Uhr abends Wehenbeginn.

11. August, $91 / 2$ Uhr früh, Blasensprung und spontane Geburt eines lebenden Mädchens. Der Austritt bis zum Nabel erfolgt rasch, dann aber 
ist ein leichter Zug nötig, um den Steiß zu entwickeln, wobei nebst dem restierenden Fruchtwasser eine reichliche Menge brauner viseider Flüssigkeit ausfließt. Abgang der normalen Placenta gleich nach der Geburt. Keine Dammverletzung.

Die Frucht zeigt einen mäbigen Ernährungszustand, ist 2600 Gramm schwer und $50 \mathrm{~cm}$ lang. Sie zeigt völlige Reife, Haare und Nägel sind vollständig entwickelt.

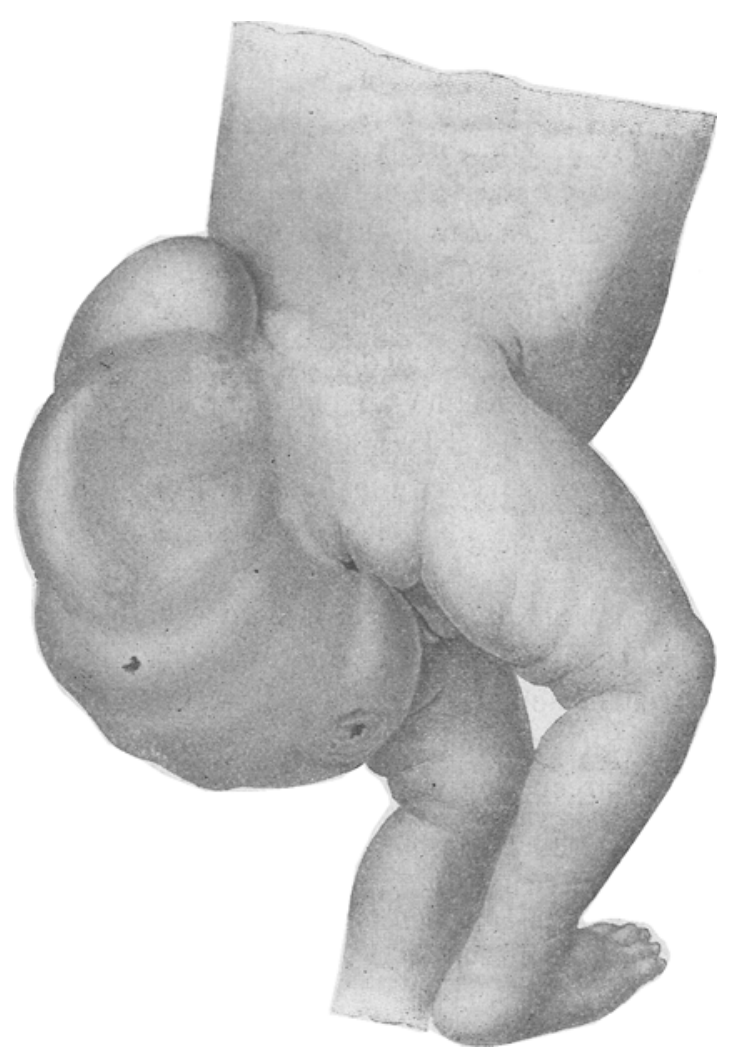

Fig. 1.

Die Maße des Kopfes betragen:

Kopfumfang:

Kopfdurchmesser:

kleiner: großer: vord. quer: $30 \mathrm{~cm} \quad 36 \mathrm{~cm} \quad 7 \mathrm{~cm}$

hint. quer: grader: groß schräg $9 \mathrm{~cm} \quad 11 \mathrm{~cm}$ klein schräg:

$81 / 2 \mathrm{~cm}$.

Im übrigen ist der Befund bis anf die zu beschreibende Mißbildung ganz normal. 
In der Sacralgegend findet sich ein weit über kindskopfgroßer Tumor, ca. $20 \mathrm{~cm}$ lang, $12 \mathrm{~cm}$ breit und $10 \mathrm{~cm}$ dick, der sich über die Mittellinie doppelt so weit nach links als nach rechts erstreckt. Die Haut darüber erseheint rechts dunkelblau durchscheinend, links normal gefärbt. Am untern Ende des Tumors findet sich eine ca. linsengroße, scharfrandige runde öffnung, etwa $5 \mathrm{~cm}$ darüber eine etwa stecknadelknopfgroße Fistel; aus beiden entleert sich spontan und auf Druck eine weißgelbliche, sehr viscide, mit verschiedengroßen corpusculären Elementen

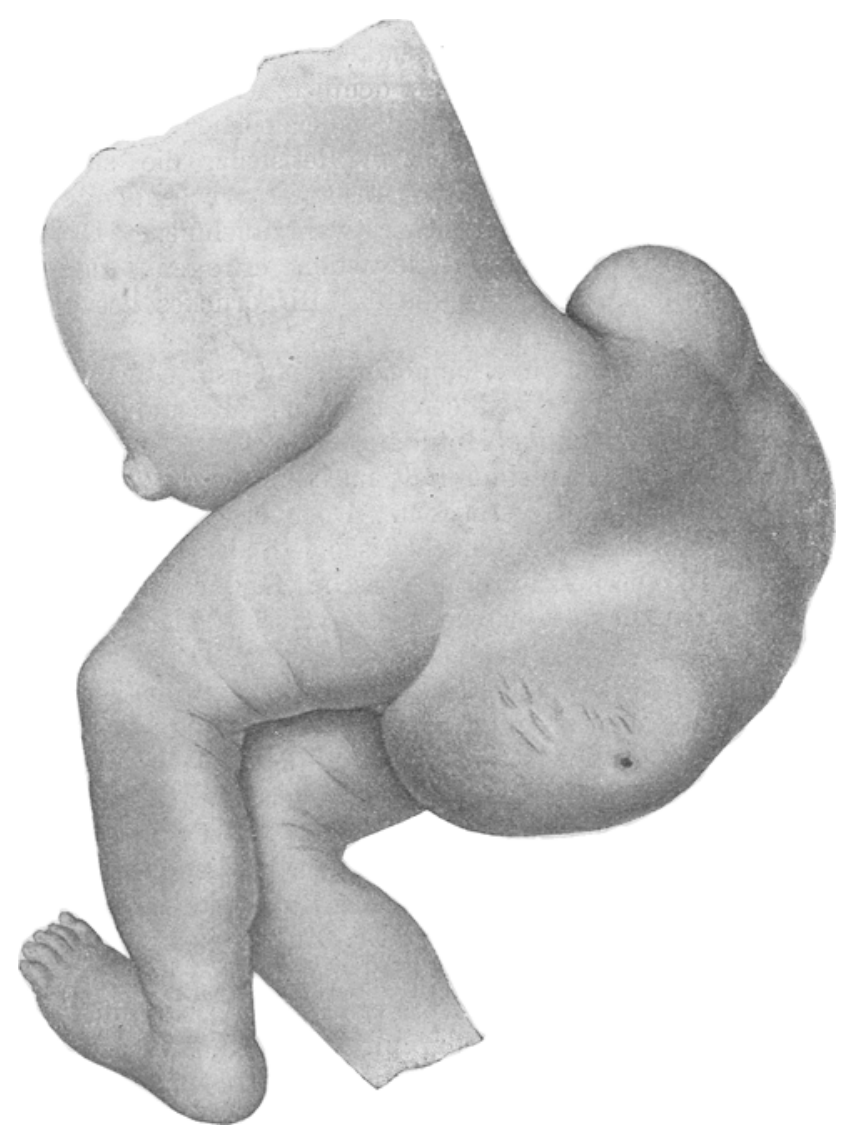

Fig. 2.

vermischte Flisssigkeit. Da bereits bei dem Austritt aus dem' Geburtskanal eine große Menge der besschriebenen Flüssigkeit abging, so ist der unterste Teil des Tumors nur mehr ein schlaffer, von Haut gebildeter Sack. Zerstreut finden sich an der Oberfläche der Geschwulst, besonders an ihrem linken unteren Anteile mehrere wechselnd große narbige, weißliche Einziehungen der bedeckenden Hautpartie. Der oberste Teil des 
Tumors ist kugelig vorgewölbt und durch eine nach unten convexe Furche deutlich abgegrenzt. Gegen den Stamm zu verschmälert sich die Geschwulst, so daß sie, wenn auch breitbasig, so doch gestielt, in das untere Stammesende übergeht.

Bei genauer Palpation erweist sich der Tumor scharf abgegrenzt von seiner Umgebung, man fühlt in den Glutaealgegend zu beiden Seiten je eine Knochenstufe (Rand des Darmbeins), von der sich der Tumor absetzt. Die Geschwulst erscheint fix und setzt sich deutlich gegen die Beckenhöble hin fort. Der obero Anteil sowie mehrere von einander getrennte Partien der unteren Hälfte zeigen exquisite Fluctuation, doch scheint keine Communication zwischen diesen mit Flüssigkeit gefüllten Hohlräumen zu bestehen. Im unteren Anteile fühlt man deutlich teils derbere, teils weichere Gebilde von knollenartiger Gestalt und verschiedener Größe; ganz distal polpiert man eine längliche knochenharte Resistenz, die sich nach unten kolbig verdickt und nach den Seiten Ausläufer zu senden scheint. Ein Defekt der Wirbelsäule ist nicht sicher wahrzunehmen. Druck auf die Geschwulst wird ohne stärkere Reflexaetion ertragen, ruft keine bemerkenswerten Symptome, wie etwa die des Hirndruckes hervor. Schreien vermehrt die Konsistenz nicht.

Die Analöffnung, vom überhängenden unteren Geschwnlstteile verdeckt, klafft nur wenig und entleert reichlich Meconium. Die unteren Extremitäten zeigen volle aktive Motilität.

Der Touchierbefund per rectum läßt nach hinten die stärkere Resistenz, die sonst das Kreuzbein gibt, vermissen. Doch läßt sich nicht mit Sicherheit ein Defekt des Beckenringes nachweisen. In der entleerten Flüssigkeit finden sich mikroskopisch zahlreiche frische Erytheocyten, teils mono-, teils polynucleäre Leukocyten, sowie viele, verschiedenartig gestaltete Epithelien.

Das durch seitliche Aufnahme gewonnene Radiogramm zeigt am unteren Anteile des Tumors einen etwa hühnereigroßen, mäßig dunklen Schattenherd und um diesen gruppiert, sowie im mittleren Teile der Geschwulst, mehrere verschieden große Herde. Die Wirbelsäule erscheint vollständig intakt, jedoch reicht sie nur mit einem kleinen Anteile in den Beckenring hinein, so daß sich der Tumor gewissermaßen in die oben von dem weit höher als normal stehenden Steißbein begrenzte Lücke hineinschiebt.

2i. August. Operation. (Doc Dr. ZuckerkandI.)

Jn leichter „Mischungs"narkose wird der Tumor circulär, allmählich von unten nach oben schichtenweise präparierend, umschnitten. Er erweist sich nur von der Haut und der darunterliegenden, schwach entwickelten auseinandergedrängten Glutaealmuskulatur bedeckt und läßt sich teils stumpf, teils mit der Schere ohne nennenswerte Blutung intakt ausschälen. Dabei besteht keinerlei Zusammenhang mit dem Wirbelkanal, der vollkommen geschlossen ist. Auffallend erscheint, daß unmittelbar unter dem Tumor das Rectum mit seiner Ampulla in seiner großen Ausdehnung gänzsich bloßliegt. Das untere Ende der Wirbelsäule (offenber hier Steißbein) ist um mehr als $5 \mathrm{~cm}$ nach oben zurück- und nach vorne gegen die hintere Rectalwand umgebogen, so daß man diesen eine Perforation drohenden, völlig soliden Teil der Wirbelsäule in der Busdehnung von etwa 2 cm abkneipt. Es werden hierauf die Bindegewebs-Muskelschichten zu 
beiden Seiten des frei liegenden Rectums lospräpariert und dureh tỉefgreifende Nähte über demselben vereinigt. Nach erfolgter Resektion der überschüssigen Hautpartie wird die Wunde durch fortlaufende Naht geschlossen und in dem unteren Wundwinkel ein Jodoformgazestreifen zur Drainage eingelegt.

Nach nahezu 2 Monaten ist die völlige Ausheilung per secundam erfolgt; das Kind befindet sich auch sonst völlig wohl und zeigt eine normale Entwickelung.

Makroskopische und histologische Beschreibung. Prosectoradjunkt Dr. Wiesel.

Der Tumor ist von unregelmäßig ovoider Gestalt. Seine Länge beträgt $18 \mathrm{~cm}$, der Umfang $12 \mathrm{~cm}$. Die Oberfläche ist teils glatt, teils von größeren, bis zu kirschengroßen Erhebungen eingenommen. Die Konsistenz des ganzen Tumors wechselt an versehiedenen Stellen; während die glatten Partien mehr derb sind, fluktnieren die mehr vorgewölbten, teils ist ihre Konsistenz bedeutend geringer als die der flachen Anteile. Auf dem größten Durchschnitte zeigt sich, daß der Tumor durehaus nicht aus einem einheitlichen Gewebe besteht. Es wechseln derbe, anscheinend aus lindegewebe bestehende Teile mit solchen, welche sich schon makroskopisch als Knochen und Knorpel erkennen lassen. Den eingangs erwähnten Vorbuchtungen entsprechen im Innern Hohlräume, die unregelmäßig begrenzt, in ihrer inneren Auskleidung zackig, von einer syrugartigen, gelblichbraunen Flüssigkeit angefüllt sind. Solche Hohlräume finden sich in größerer Anzahl durch die ganze Dicke des Tumors verteilt. Ferner zeigen sich an Schnitten durch den Tumor in verschiedenen Höhen streifenförmige Pigmentanhäufungen, sowie Inseln von Parenchymen, die schon makroskopisch eine Organdiagnose gestatten. Z. B. kleine, hellergroße Partien von Leber, ferner eine wohl charakterisierte, ohne weiteres erkennbare Nebenniere, eine Speicheldrüse u. s. w.

An der äußeren Peripherie des Tumors läuft eine schmale Rinne fast rings um die ganze Circumferenz, deren beide Wände von Haut ausgekleidet sind. Es läßt sich also sehon auf Grund des makroskopischen Befundes der Tumor als Teratom diagnostizieren.

Es wurden nun ans verschiedenen Partien des Tumors Stücke ent. nommen, um sie der histologischen Untersuchung zuzuführen. Diese Stücke, verschieden groß gemacht, wurden in Celloidin eingebettet und geschnitten. Die histologische Untersuchung führte zu dem Ergebnis, daß Derivate sämtlicher $3 \mathrm{Keimblätter} \mathrm{vorhanden} \mathrm{waren.}$

Ich beginne mit den ektodermalen Gebilden.

Vom Großhirnbläschen sind größere Partien zur Entwickelung gelangt; man findet an einzelnen Schnitten Stellen, die der Adergeflechtsfurche entsprechen, ferner Gebilde, die als Ammonshörner zu bezeichnen sind.

Vom Zwischenhirnbläschen findet sich der III. Ventrikel, das Infundibulum und das vordere Adergeflecht. Die aus dem hintersten Teile der Deckplatte des Zwischenhirnbläschens sieh entwickelnde Zirbeldrüse konnte nicht aufgefunden werden.

Vom Mittelhirnbläschen findet sich der Aquaeductus Sylvii und die Vierhügel. 
Vom vierten Hirnbläschen resp. seinen Derivaten konne nichts gefunden werdeu; ebensowenig vom 5. Hirnbläschen. Rückenmark fehlte ebenfalls.

Vom Auge waren beträchtliche Teile zur Ausbildung gelangt und zwar in paariger Anlage. Es fand sich in vollkommener Entwicklung: Cornea, Sklera, der Ciliarkörper, ferner die Pars iridica retinae, sowie Chorioidealepithel; die oben erwähnten Pigmentanhäufungen gehören größtenteils dem Binnenpigment des Auges an. Eine fernere Differenzierung der Retinalelemente konnte nicht erzielt werden. Ein Sehnerv war nicht zu finden, ebenso fehlten auch die übrigen Hirnnerven sowie Augenlider und Tränenorgane. Vom Geruchs- und Gehörorgan fanden sich keinerlei mit Sicherheit zu diagnostizierende Elemente.

Die Haut war in allen ihren Abschnitten teils in Form des oben erwähnten Ringes, teils in Form von verschieden großen Inseln vorhanden und gut ausgebildet. Sowohl Corium als Epidermis zeigten durchaus normalen Bau.

Von Anhangsgebilden der Haut ließen sich Nägel nicht auffinden, Hare waren in ihrer Anlage als Haarkeime und Haarpapillen vorhanden, freie Haare fehlten; dagegen fanden sich reichlich Talg- und Schweißdrü sen.

An Hautstellen, die zu Extremitätenanlagen gehörend gedeutet werden konnten, fanden sich vereinzelte, aber wohl ausgebildete Tastkörperchen.

Die Organe des mittleren und inneren Keimblattes waren reichlich vertreten, allerdings nicht in Form von zusammenhängenden Organsystemen, wie beispielsweise der Darmkanal, sondern wiederum größtenteils in Einzelform angeordnet, ohne jegliche bestimmtere Topographie, seltener in größeren Verbänden.

Willkürliche Muskulatur war in großer Menge auffindbar, besonders an jenen Stellen, welche eventuell als Extremitätenanlage angesprochen werden konnten, aber auch außerdem in Form breiterer Platten. (Rumpf- und Thoraxmuskeln?)

Bleibende Niere war in einem kleinen Exemplar vorhanden; allerdings ohne jeglichen Zusammenhang mit einem nach seiner epithelsalen Auskleidung vielleicht Harnblase zu deutenden Hohlraum.

Ureteren fehlten, ebenso eine jegliche Andeutung einer Geschlechtsdrüse oder deren Ausführungswege.

Trotz Untersuchung sämtlicher Höhen des Tumors fand sich nichts, was mit Sicherheit als Geschlechtsdrüse bezeichnet werden konnte. Selbstverständlich fand sich auch kein äußeres Genital oder eine Kloake.

Vollständig ausgebildet in ihren sämtlichen Kindenpartien war eine schon makroskupisch gut erkennbare Nebenni ere; ihr chromaffiner Abschnitt fehlte dagegen, ebenso wie eine andere Anlage des sympathischen Nervensystems.

Um auf die Niere zurückzukommen, so sei nur kurz bemerkt, daß sie sowohl in der Rinden-als Markanlage wohl differenziert war. Sie endete anscheinend blind in einer Art von in sich geschlossenem Nierenbecken. Harnsäureinfarcte waren nicht vorhanden. 
Vom inneren Keimblatt fanden sich eine Anzahl von Schlundspaltenderivaten. Die Mundbucht allerdings war nicht zur Entwicklung gelangt, der Darmkanal jedoch war in seinen einzelnen Abschnitten reichlich repräsentiert. Vor allem erwiesen sich die schon obenerwähnten, mit gallertartigem Inhalt erfüllten Hohlräume als zu ihnen gehörend. Es fanden sich Stellen mit Pflasterepithel des Oesophagus, ferner solche mit Drüsenschläuchen, wie sie dem Magen entsprechen, Dünndarm mit Zotten und Brunnersehe Drüsen des Duodenums, Dickdarm, aber alles wahllos durcheinander gewürfelt ohne jeglichen Zusammenhang. Von Anhangdrüsen des Darmes fanden sich: Leber, Pankreas, Speiche ldrüsen. Dagegen verdient das fast völlige Fehlen von lymphoiden Gew ebe hier hervorgehoben zu werden, so fehlte beispielsweise jegliches Organ, das als Milz hätte gedeutet werden können. Auch der lymphatische Apparat des Darmes war äußerst spärlich. Lungen fehlten.

An Organen des Mesenchyms fand sich das Herz in Form eines einkammerigen Schlauches ohne deutliche Vorhöfe und Klappenapparate. Größere Gefäße zogen kreuz und quer durch den Tumor, ohnn eine bestimmte Topographie erkennen zu lassen.

$\mathrm{Kn}$ ochen waren in Form kurzer Röhren und kleiner Belegknochen vorhanden. Doch war keiner deutlich als Teil der Extremitäten oder Wirbelsäule zu diagnostizieren. Die vorhandenen Knochenanlagen zeigten alle Stadien der Ossifikation.

Auch waren alle Formen von Stützubstanz vertreten. Zahnanlagen waren nicht auffindbar.

Es war unmöglich, eine bestimmte Topographie anzugeben. Wirr und regellos lagen die heterogensten Organanlagen nebeneinander. Nirgends fand sich ein morphologischer oder physiologischer Zusammenhang.

Angeborene Sacraltumoren sind ein nicht allzu häufiges Vorkommnis. Calbet gibt 1 auf 34582 Geburten an. Das weibliche Geschlecht ist im allgemeinen öfter betroffen. Von 203 Fällen Calbets fallen 126 auf Mädchen, nur 60 auf Knaben.

Kiderlen fand das Verhältnis der beiden Geschlechter wie 1:4. In Gerhardts Handbuch allein fand ich die Ansicht vertreten, dab das männliche Geschlecht häufiger als das weibliche mit Sacraltumoren behaftet sei. Relativ häufig ist diese Mißbildung mit Hydramnios vergesellschaftet. So fand Condére diese Komplikation in 29 Fällen 8 mal, Calbet in 107 Fällen nur 11 mal. Die Geburten finden bei Früchten mit Steißtumoren oft vor dem normalen Sichwangerschaftsende statt. Calbet hat unter 117 Fällen nur 10 ausgetragene Früchte und 19 Frühgeburten, Kiderlen unter 119 Fällen 10 Frühgeburten. Die Unterbrechung der Schwangerschaft findet meist im 6 Monate statt.

Die meisten Kinder mit Sacraltumoren kommen tot zur Welt. Calbet fand in 107 Fällen 211 totgeborene Früchte, wovon 13 Frühgeburten und 7 reif waren, 7 Kinder starben während der Geburt. 
Dazu bemerkt Linser, daß die Hälfte der Kinder mit Steißgeschwülsten während der Geburt oder im ersten Lebensjahre stirbt.

Die Geburten verlaufen gewöhnlich normal. Daß die Kinder meist in Kopflage geboren werden, ist daraus zu erklären, daß oft Frühgeburten vorkommen und die Tumoren dann meist Faustgröße nicht überschreiten. Sperling berichtet über 2 kindskopfgroße Tumoren. Leclerk über einen $950 \mathrm{~g}$ schweren. Darnach ist der vorliegende Tumor wohl als einer der größten bisher beobachteten anzusehen. Hohl erwähnt, daß in 40 Fällen 22 Geburten spontan verliefen und $1 \delta$ mal Kunsthilfe nötig war. Aber auch unter den Spontangeburten waren 6 sehr schwer, in 3 Fällen barst die Geschwulst. Auch in unserem Falle lälit sich der rasche, fast mühelose Durchtritt des reifen Kindes nur dadurch erklären, daß der cystische Teil des Tumors spontan zerriß und um ein Erhebliches ver. kleinert wurde. Braun e fand bei 79 Fällen nur 22 erschwerte Geburten.

Das Geburtshindernis tritt, da es sich, wie erwähnt, meist um Schädellagen handelt, erst nach der Geburt des Kopfes und Rumpfes auf. Die Ursache kann in dem oft gleichzeitig mitvorhandenen H ydramnios liegen. Es genügen dann einige kräftige Traktionen, das bis zum Nabel geborene Kind völlig zu extrahieren. Oft aber bereitet die Größe des Tumors ein unüberwindliches Hindernis, oder die Gesehwulst hackt sich unter der Symphyse fest. Tritt der letztere Fall ein, so kann man dadurch, daß man den Tumor durch Rotation in die Kreuzbeinhöhle zu brıngen sucht, eine leichte Entwicklung anbahnen. Calbet, Tarnier und $K \ddot{~ u ̈ s t e r ~ r a t e n, ~ d i e ~ F u ̈ ß e ~ n e b e n ~ d e m ~}$ Tumor herabzubolen und kräftig anzuziehen. Da die Frucht bei längerem Verweilen in dieser Lage meist abstirbt und Eile nottut, so kam es wiederholt bei energischeren Bemiibungen zu Einrissen in den Tumor und Abfluß von Flïssigkeit, worauf, we in unserem Fall, die Geburt glatt von statten ging. Solche Fälle erwähnen Calbet, Glogner und Martin. Bei Beumer rib der Tumor glatt ab. Es wird deshalb von manchen Autoren eine direkte Punktion des nachfolgenden Tumors empfohlen. Steißlagen erschweren die Geburt nicht allzu oft; es genügt dann ein kräftiger Zug am Tumor oder die Punktion desselben, um die Frucht zu entwickeln. Doch ist die Diagnose in solchen Fällen oft recht schwer, und es sind wiederholt Verwechslungen mit der Fruchtblase oder Placenta praevia vorgekommen. Besonders eingreifende Operationen erscheinen nach dem Gesagten selten nötig, doch sind auch Fälle beschrieben, in denen es zur Embryotomie i. e. zur Abtrennung des Tumors im Uterus kam (Uth möller). 
Kommen wir auf die Genese des vorliegenden Tumors zurück, so kann es wohl auch für den eingefleischtesten Anhänger der monogerminalen Theorie kaum einen Zweifel unterliegen, daß es sich hier um eine $\mathbf{z}$ weite Keimanlage handelt. Es gibt nur äußerst wenige unter den bisher veröffentlichten Fällen, die mit Ausnahme des Respirationstraktes und der Genitalsphäre eine so reichbaltige, fast lückenlose und eindeutige Aufzählung der vorhandenen Organanlagen zuließen. Es sind Derivate sämtlicher Keimblätter vorbanden. Es ist nicht nur der Darm mit seinen Anhangsgebilden zur Entwicklung gelangt, sodaß 3 auch der hochgestellten Forderung Nasses genügt ist, indem durchaus nicht ausschließlich die dem hinteren Stammesende des Embryo entsprechenden Organe den Tumor zusammensetzen. Aber auch die nervösen Elemente treten nicht so sehr in den Vordergrund, wie es Wieting und Engelmann für die Allgemeinheit der Embryome annehmen. Der Digestionstrakt ist in allen seinen Abschnitten vertreten, und auch seine größeren Anhangsdrüsen, wie Leber, Pankreas und Speicheldrüsen sind deutlich entwickelt. Daß auch das A uge in nahezu allen Bestandteilen, ferner die vollausgebildete Niere, das Herz, die Andeutung von Extremitätenanlagen durch die Tastkörperchen und sämtliche Arten des Stützgewebes in allen Stadien ihrer Entwicklung aufgefunden war, muf wohl als ein nicht allzu häufiges Vorkommnis bezeichnet werden. Ganz besonders interessant und bisher wenig oder gar nicht beobachtet ist das Vorhandensein der gut ausgebildeten Rindensubstanz der Nebenniere. Nur von den Respirationsorganen und dem Genitale waren trotz genauen Durchforschens des Tumors keine Anlagen zu finden.

Gehen wir nun auf die eigentliche Pathogenese dieser Tumoren und speziell des vorliegenden näher ein, so ergibt sich folgendes.

Sobotta hat am Ei von Mäusen den Nachweis erbracht, daß jedes während der Reifezeit abgestoßene Richtungskörperchen ein abortives befurchtungsfähiges $\mathrm{Ej}$ darstelle. Daraus folgern die meisten Autoren auf einen ähnlichen Vorgang beim Menschen. Diese Polzelle kann sich in derselben Weise wie die Mutterzelle entwickeln oder in der Entwicklung weit zurückbleiben. Daraus ergibt sich dann die mehr oder minder große Verschiedenheit in der Vollkommenbeit der Doppelbildung. Die zurückgebliebenen, vom normalen Embryo umwachsenen bilden die foetalen Inklusionen. In den meisten Fällen erfolgt die Verwachsung, wie K ol is k o hervorhebt, am Schwanzende. Es sei an dieser Stelle in Parenthese bemerkt, daß nach 
Kolisko, der sich um die Aufhellung dieser Frage sehr verdient gemacht bat, für alle sacralen Embryome die gestreckte Stellung des Kreuzbeins und die nach binten verlaufende Krümmung des Steißbeins ein gemeinsames wichtiges diagnostisches Merkmal sei; für das Kreuzbein traf es in unserem Fall wohl zu, nur war dasselbe um ein gutes Stïck aus dem Beckenring nach oben hinausgeschoben oder besser gesagt, durch den Tumor verhindert, sich dem knöchernen Ring ganz einzufügen; das Steißbein war nach vorin gekrümmt, weshalb es entfernt werden mußte.

Auch eine zweite Möglichkeit des Entwicklungsmechanismus der Embryome wurde von Bonnet angegeben. Darnach kann es in einer sonst normal angelegten Keimblase zu einer Überproduktion von Furchungskugeln kommen, die nicht zum Aufbau des Organismus verwendet, aber früher oder später sich weiter entwickeln. Wenn sich eine solche Blastomere frühzeitig loslöst, so wird es auch zu einer weitgehenden Differenzierung kommen, während in späteren Stadien nur ein oder zwei Keimblätter vertreten sein werden. Es berühren also alle diese Tumoren auf einer einheitlichen Grundlage, und der Unterschied ist nur ein gradueller. Hagen hat in Beipflichtung dieser Anschauungen folgende übersichtliche Einteilung angegeben :

I. Embryome, die aus einer befruchteten Polzelle hervorgegangen sind. (Unvollkommene Doppelbildungen, ein Teil der foetalen Inclusionen.)

II. Embryome, die aus der Überproduktion von Furchungskugeln enstanden sind:

a) Solche, die Ablömmlinge aller 3 Keimblätter enthalten ('Tridermome)

Teratome im engeren Sinne (zusammengesetzte Dermoide) der Rest der bisher als foetale Inclusionen bezeichneten Tumoren.

b) Solche, die nur aus Abkömmlingen zweier Keimblätter bestehen (Bidermome) (zusammengesetzte Dermoide).

c) Solche, die nur Abkömmlinge eines, des äußeren Keimblattes enthalten: einfachen Dermoide.

Wenn ich die Gruppe II c ausschalte, über deren Entstehung und Zugehörigkeit ich mich eingangs dieser Zeilen äußerte, so erscheinen mir alle Embryome nach dieser Einteilung obne Zwang unter einen einheitlichen Gesichtspunkt gebracht, der wohl ziemlich stichhaltig ist. 
Es erübrigt nun noch, eine einigermaßen plausible Unterscheidung zu treffen zwischen den aus 2 Keimanlagen entstandenen Embryomen und solchen, die aus überzähligen Furchungskugeln hervorgegangen sind. Wilms bat die Meinung ausgesprochen, daß alle Embryome, die Produkte beider Körperhälften gebildet haben, aus einem A bortivei entstanden seien. Dafür liegt nach $\mathrm{Hagen}$ keln zwingender Grund vor, der höchstens für offenkundige Halbseitigkeit die Annahme der Genese aus einer Furchungskugel für berechtigt hält.

Mehr als diese Momente dürften wohl der Grad der Differenzierung und die Zweckmäßigkeit in der ganzen Anlage Anhaltspunkte für eine eben immer nur wahrscheinliche Entscheidung dieser Frage geben. Falls sich ein planmäßiger Aufbau, eine hervorstechende Ähnlichkeit mit dem Embryo selbst', eine "gewollte Anordnung zu Organsystemen" nachweisen läßt, so wäre die Genese aus einem Abortivei zu erklären. Bei einem wirren, regellosen Durch. einander der verschiedensten Organteile ohne bestimmte Topographie könnt man geneigt sein, die Weiterentwicklung einer abgestoßenen Furchungskugel als Entstehungsursache anzunehmen. Auch für unseren Fall wäre diese letztere Erklärungsweise am Platze, und er fände in der Einteilung $\mathrm{Hagens}$ in der Gruppe II a seine angemessene Stelle.

\section{Literaturverzeichnis.}

Borst, Die angeborenen Geschwälste der Sacralregion. Centralbl. f. allg. Pathol. u. path. Anatom. IX 1898.

Calbet, Contribution à l'étude des tumeurs congénitales d'origine parasitaire de la region sacro-coccygienne. Paris. 1893.

Engelmann, Beitrag zur Kenntnis der Sacraltumoren. Arch. f. klin. Chir. 72. 1904.

Gerhardts, Handbuch der Kinderkrankh. VI. a.

Giani, Contributo allo studio dei teratomi sacralı. Clin. chir. 1903. 11. Graff, Deutsche Zeirschr. f. Chir. 1904.

Hagen, Beitrüge z. klin. Chir. 1904.

Hoffmann, Ein Beitrag zu den angeborenen Sacralgeschwülsten. J. D. Leipzig. 1904.

Hoppe, Beitrag zur Lehre von den angeborenen Kreuzsteißbeingeschwülsten. D. Zeitschr. f. Chir. 66. 1903.

Kiderlen, D. Zeitschr. f. Chir. 52. 1899.

Kolisk o, Wien. klin. Woch. 1890. p. 389.

I d e m, ibidem. 1894. p. 391,

Linser, Beitr. z. klin. Chir. 29. 1900.

Nasse, Beitrag zur Genese der sacrococcyg. Teratome. Arch. f. klin. Chir. 45. Pflanz, Zeitschr. f. Heilkunde. 1896. 
382 XVIII. Frank, Zur Kenntnis der congenitalen Sacraltumoren.

Preindlsberger, Zeitschr. f. Heilkunde. 1903.

Sänger, Arch. f. Gyn. 37. 1890.

Steinthal, Handb. d. pract. Chir. VI.

Stolper, Die angeborenen Geschwülste der Kreuzsteißbeingegend. D. Zeitschr. f. Chir. 50. 1899.

Tillmanns, Deutsche med. Woch. 1904. 17.

Uthmöller, Über Geburten bei SteiBtumoren. Monatsschr. f. Geburtsh. u. Gyn. 1904.

Wilms, D. Arch. f. klin. Med. 55.

I d em, Die Mischgeschwülste. Berlin. 1899/1902. 